\title{
Les styles d'apprentissage des étudiants de la faculté de médecine de Sousse (Tunisie)
}

\author{
Learning styles of medical student in the faculty of medicine \\ of Sousse (Tunisia)
}

\author{
Meriam EL GHARDALLOU ${ }^{1,2}$, Thouraya AJMI'1,2, Iheb BOUGMIZA ${ }^{1,2}$, \\ Chekib ZEDINI ${ }^{1,2}$, Manel MELLOULI ${ }^{1,2}$, Ali MTIRAOUI ${ }^{1,2}$. \\ ${ }^{1}$ Département de Médecine familiale et communautaire, Faculté de médecine de Sousse, Université de Sousse \\ ${ }^{2}$ Unité de recherche « Pratique Médicale Ambulatoire » PMA 01/UR/08-15
}

Manuscrit reçu le 16 mai 2012 ; commentaires éditoriaux formulés aux auteurs le 10 mars 2103 et le 8 août 2013 ; accepté pour publication le 15 août 2013

\begin{abstract}
Mots-clés
Étudiant ; médecine ; styled'apprentissage ; ISALEM 97

Keywords Student; medicine; learning styles; ISALEM-97

Résumé - Contexte : Le style d'apprentissage semble jouer un rôle dans le choix de l'approche d'apprentissage prometteuse de succès. Ainsi, il pourrait être opportun que les éducateurs cherchent à identifier les styles d'apprentissages des étudiants et à y adapter leurs dispositifs pédagogiques. But : Décrire les styles d'apprentissage des étudiants de la faculté de médecine de Sousse (Tunisie). Sujets et méthodes : Une étude descriptive de nature transversale a été menée auprès de cinq promotions d'étudiants de la Faculté de médecine de Sousse au cours de l'année universitaire 2009-2010. L'instrument de mesure utilisé était l'Inventaires des Styles d'Apprentissage du Laboratoire d'Enseignement Multimédia -ISALEM 97, développé par l'Université de Liège, qui suggère l'existence de quatre styles d'apprentissage. Résultats : Huit cent cinquante six étudiants ont participé à l'étude pour un total de 1043 (taux de réponse : $82 \%$ ). Le style prédominant était le style intuitif-pragmatique dans $40 \%$ des cas, suivi du style méthodique- pragmatique dans $28 \%$ des cas. La dimension de transformation de l'expérience différait significativement selon le sexe. L'analyse en composantes principales confirme la structure bidimensionnelle de l'ISALEM 97. Les coefficients de Cronbach étaient $\geq 0,72$. Conclusion : Les étudiants de la faculté de médecine de Sousse ont plutôt un style intuitif-pragmatique ; ils recourent à l'intuition pour la saisie des concepts et ont tendance à les appliquer dans de nouvelles expériences pour résoudre les problèmes. Ce profil ne change pas tout au long des années d'études médicales mais différent légèrement en fonction du sexe.

Abstract - Context: Learning style appears to play a significant role in choosing a successful learning approach. The teachers' role is to identify and adapt the teaching techniques to their student's styles. Goal: Describing the learning styles of medical students at the faculty of medicine of Sousse (Tunisia). Subjects and methods: A descriptive cross-sectional study was conducted among five cohorts of students from the faculty of medicine of Sousse during
\end{abstract}


the 2009-2010 academic year. The ISALEM-97 test developed by the University of Liège was used. It divided individual learning styles into four types. Results: On a total of 1.043 students, 856 took part in the study (overall response $82 \%$ ). The intuitive pragmatic learning style was prevalent in $40 \%$ of cases, followed by the methodical pragmatic style in $28 \%$ of cases. The transformation dimension of the experience varied significantly depending on gender. The factorial analysis of the main components confirms the two-dimensional structure of the ISALEM-97 test. The Cronbach coefficient was $\geq 0.72$. Conclusion: Students of the faculty of medicine of Sousse have a more intuitive pragmatic learning style: they use intuition to grasp concepts and tend to apply it in new experiences to resolve problems. The profile does not change over the length of medical studies but varies slightly depending on gender.

\section{Introduction}

Contexte académique de la Faculté de médecine de Sousse

Prenant en compte les changements majeurs survenant dans son environnement et afin d'adapter les compétences de ses diplômés aux nouvelles exigences de la société et aux nouveaux besoins de santé et de bien être, la Faculté de médecine de Sousse (FMS) (Tunisie) a entrepris en 2003 une initiative d'auto évaluation, suivie en 2005 par une évaluation externe conduite par la Conférence internationale des doyens et des facultés de médecine d'expression française (CIDMEF) ${ }^{[1]}$. A l'issue de cette réflexion critique approfondie sur l'ensemble de l'institution (mission, enseignement, étudiants, ressources, direction, ...), différentes recommandations ont été formulées, parmi lesquelles celle qui invitait à concevoir une reforme pédagogique globale a été jugée importante.

Le curriculum en place comporte d'une part, un programme d'enseignement théorique, réparti en deux cycles : un premier se déroulant sur deux années, dédié aux sciences cliniques dites fondamentales, à la médecine communautaire, à la sémiologie et à plusieurs formations générales (anglais, secourisme, psychiatrie, histoire de la médecine); un deuxième se déroulant sur trois années et comprenant des certificats intégrés et des certificats multi modulaires. Il comporte, d'autre part, des stages cliniques d'externat, organisés de la troisième à la cinquième année durant les matinées du lundi au vendredi, auxquels font suite deux années consacrées en totalité à la pratique clinique (les stages d'internat).

L'analyse globale de ce dispositif curriculaire a révélé de nombreux problèmes tels que l'absence de cadre de référence pédagogique et d'objectifs institutionnels explicites, des activités d'enseignement de nature essentiellement magistrale ou encore l'absence de coordination entre l'enseignement théorique et les stages pratiques ${ }^{[1]}$. En conséquence, un projet de réforme pédagogique des études médicales a été développé, qui devrait orienter progressivement les dispositifs pédagogiques en place à la faculté vers des méthodes d'enseignement interactives permettant aux étudiants de jouer un rôle actif et de développer des habitudes d'auto apprentissage et d'auto évaluation $^{[1]}$.

Dans cette perspective, différentes actions doivent être développées; elles concernent à la fois le contexte éducationnel dans lequel l'étudiant évolue (méthodes d'enseignement, programme à enseigner, ...) et les activités d'apprentissage de l'étudiant luimême. Ainsi, l'identification des styles d'apprentissage des étudiants en médecine est potentiellement d'une grande pertinence pour les éducateurs, les administrateurs et les étudiants eux-mêmes ${ }^{[2]}$, les enjeux se situant à un double niveau : d'un côté, il s'agit pour les enseignants d'examiner la nécessité d'adapter leurs interventions pédagogiques pour mieux prendre en compte les styles d'apprentissage des étudiants ; de l'autre, il s'agit de discuter l'opportunité d'intervenir directement ou indirectement auprès des étudiants pour qu'ils prennent conscience de leurs styles d'apprentissage et qu'ils utilisent les techniques d'apprentissage les mieux adaptées à leurs 
styles, ce qui améliore leur satisfaction en tant qu'apprenants ${ }^{[3]}$.

C'est dans le cadre de cette dynamique qu'il a paru nécessaire de se préoccuper du style d'apprentissage des étudiants de la FMS, afin de mieux orienter le processus d'amélioration de la formation médicale au sein de cette institution.

\section{Cadre conceptuel}

«Inspiré au début par la théorie du traitement de l'information [...] et progressivement influencé par les recherches sur la motivation et la métacognition, Biggs a développé le concept d'approche d'apprentissage ${ }^{[4]}$. Selon cet auteur ${ }^{[5]}$, l'approche d'apprentissage comporte trois dimensions, respectivement en profondeur, de surface et stratégique ${ }^{[4]}$. L'apprentissage est vu comme étant un système ouvert dans lequel il existe une interaction complexe de divers éléments. Selon la perception qu'il a de ses caractéristiques individuelles d'une part (style d'apprentissage, sentiment de compétence, connaissances antérieures, buts d'apprentissage, ...) et des caractéristiques du contexte académique d'autre part (l'institution, méthodes d'enseignement, programme éducatif, enseignant, moyens d'évaluation, ...), l'étudiant adoptera une approche plutôt en profondeur ou plutôt en surface ${ }^{[4]}$. A cet égard, les enseignants doivent privilégier en général les curricula qui favorisent une approche en profondeur, d'autant que plusieurs études ont conclu à l'existence d'une corrélation positive entre ce type d'approche et le succès scolaire ${ }^{[6]}$. Il est ainsi possible de postuler que le style d'apprentissage de l'individu joue un rôle dans le choix de l'approche d'apprentissage et il devient dès lors légitime que les éducateurs cherchent à identifier et prendre en compte les différents styles d'apprentissage des étudiants, éventuellement en les faisant évoluer, pour favoriser au mieux le recours à l'approche en profondeur, plus prometteuse de succès.

D'un point de vue historique, la notion de style d'apprentissage a pris naissance à partir de la notion de style cognitif, défini par Allport ${ }^{[5]}$, psychologue de la personnalité, comme étant «l'ensemble à la fois cognitif et affectif, de traits personnels (modes fondamentaux de penser et d'agir), qui orientent ses perceptions, ses images et ses jugements à propos de son mode personnel ». Ainsi, selon cet auteur, chaque action d'une personne comporte un aspect adaptatif et un aspect expressif.

A partir des années soixante, et afin de respecter les différences individuelles des apprenants, les chercheurs ont développé des outils conceptuels et des pratiques pour mesurer les caractéristiques des étudiants en terme d'apprentissage plutôt qu'en termes généraux du fonctionnement cognitif. Ceci a donné lieu à la notion de style d'apprentissage, plus spécifique et ayant une visée plus pédagogique que celle de style cognitif ${ }^{[8]}$. Cette nouvelle notion apparue alors au cours des années 70, a fait l'objet de plusieurs définitions de la part des chercheurs, chacune se caractérisant par une classification particulière des styles d'apprentissage. Chevier et al. ${ }^{[8]}$, présentent six principaux cadres de référence permettant d'analyser les différentes typologies de styles d'apprentissage, respectivement : l'environnement pédagogique, les modalités d'encodage et de représentation, les modalités de traitement de l'information, l' apprentissage expérientiel, une théorie de la personnalité, des modèles mixtes. Ces mêmes auteurs considèrent que la typologie de styles d'apprentissage développée par Kolb est la première à s'être adossée à un modèle d'apprentissage, en l'occurrence l'apprentissage expérientiel ${ }^{[8]}$. Selon $\mathrm{Kolb}^{[9]}$, l' apprentissage est le processus pour lequel le savoir est créé par la transformation de l'expérience. Cette théorie se différencie à la fois d'une part, des théories rationalistes et autres théories cognitives de l'apprentissage, qui donnent un rôle essentiel à l'acquisition, la manipulation et au souvenir des symboles abstraits et d'autre part, des théories comportementalistes de l'apprentissage (béhaviorisme $)^{[10]}$. Par ailleurs, d' autres auteurs rapprochent cette conception de l'apprentissage de celle issue de la perspective constructiviste, qui met l'accent sur le fait que les connaissances se construisent dans un processus d'interaction entre le sujet et son environnement ${ }^{[10]}$. Ainsi, selon la conception même de $\mathrm{Kolb}^{[9]}$, la perspective de l'apprentissage expérientiel ne constitue pas une nouvelle alternative aux théories cognitives et comportementales mais propose une perspective holistique intégrative de l'apprentissage, 
combinant expérience, perception, cognition et comportement. D'une façon globale, elle postule que l'apprentissage est un processus majeur de l'adaptation humaine, apparaissant dans tous les cadres humains (école, travail, ...), se développant à toutes les étapes de la vie, selon un continuum performance (adaptation à court terme) - apprentissage - développement adaptif à l'environnement (adaptation tout au long de la vie à toutes les situations), grâce à des processus qui ne varient que par leur degré d'extension dans le temps et dans l'espace ${ }^{[9]}$

Une telle perspective s' avère applicable à certains domaines qui nécessitent une adaptation continue et globale, comme c'est le cas au cours des études médicales où l'apprenant doit d'abord développer ses compétences lors des différentes phases de sa formation puis assurer son développement professionnel continu en intégrant la masse d'informations et de savoirs nouveaux générés par les progrès scientifiques. En effet, les apprenants doivent être capables de créer des concepts qui intègrent leurs observations dans des théories solides et ils doivent être capables d'utiliser leurs théories pour prendre des décisions et résoudre des problèmes. En somme, l'apprentissage requiert des capacités correspondant aux deux dimensions primaires du processus d'apprentissage : la première représentée par l'expérimentation concrète des événements afin de conceptualiser pour autrui, et la seconde est la dimension reliant l'expérimentation active à l'observation réfléchie ${ }^{[6]}$.

Le cycle d'apprentissage expérientiel comporte quatre phases (Figure 1): l'expérience concrète (EC), l'observation réfléchie (OR), l'abstraction conceptuelle (AC) et l'expérimentation active (EA). Selon $\mathrm{Kolb}^{[9]}$, l'apprentissage significatif ne survient que lorsque la personne a réalisé les quatre moments du cycle par rapport à un objet d'apprentissage donné.

En s'adossant à ce modèle du processus d'apprentissage expérientiel en quatre étapes, Kolb a fait l'hypothèse de quatre modes d'adaptation (concret, réfléchi, abstrait et actif), qui, combinés deux à deux, forment quatre styles d'apprentissage : le style divergent (concret-réfléchi) ; le style assimilateur (réfléchi-abstrait ; le style convergent (abstrait-actif) et le style accommodateur (concret-actif).

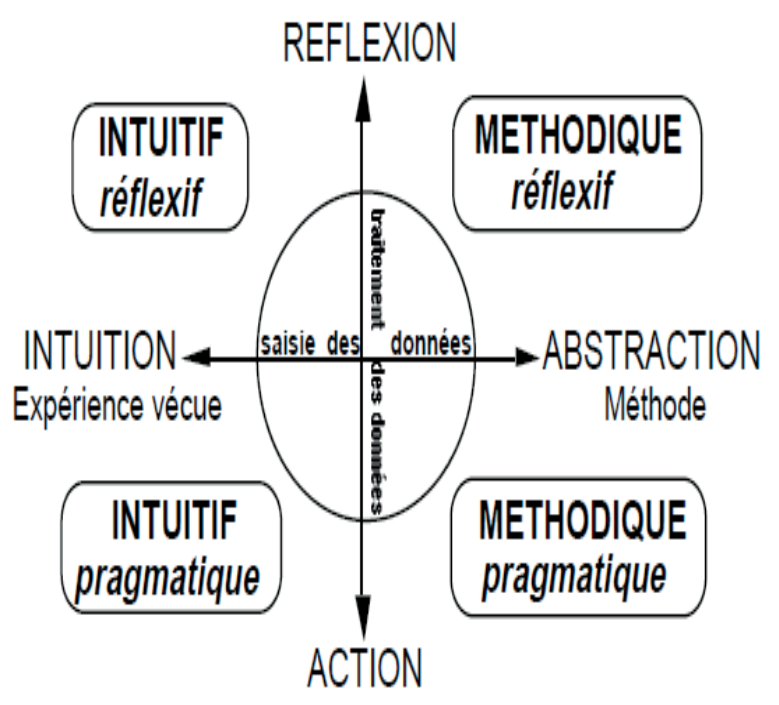

Fig. 1. Le cycle d'apprentissage expérientiel selon David Kolb.

\section{Le style intuitif réflexif (divergent)}

Il caractérise une personne qui aime examiner une situation sous différents angles avant de s'y engager. Elle privilégie un mode d'observation et une réflexion pour s'assurer de la validité de l'action à entreprendre. Elle est particulièrement douée pour l'imagination, la compréhension des gens et l'identification des problèmes. Par contre, sa minutie peut être à son désavantage si elle tarde inutilement à prendre des décisions. Dans certaines circonstances, l'action doit être entreprise promptement et une réflexion prolongée comporte plus de risque qu'une intervention rapide.

\section{Le style méthodique réflexif (assimilateur)}

Il reflète la préférence d'une personne pour l'organisation et l'intégration de ses observations à des systèmes de référence. Rationnelle, cette personne conçoit le niveau d'abstraction qui sous-tend toute action et le privilégie. La rigueur et la validité des théories forment son principal domaine d'intérêt. Elle est douée pour la planification, la création des «modèles scientifiques », la définition des problèmes et le développement des théories. 


\section{Le style intuitif pragmatique (accommodateur)}

Il se réfère à une personne ouverte d'esprit et flexible. Elle s'engage totalement et sans idées préconçues dans des expériences nouvelles. Elle apprend par l'action et s'approprie ainsi la connaissance. Elle sait bien relever le défi de la mise sur pied d'un projet ; par contre, elle s'avère moins intéressée à sa consolidation à long terme.

\section{Le style méthodique pragmatique (convergent)}

Une personne de style méthodique s'intéresse à la mise en application et à la généralisation des idées, des théories, des techniques. Elle en évalue rapidement l'efficacité et accomplit les taches nécessaires pour les expérimenter. Elle choisit des moyens judicieux qui lui permettent de mettre en pratique ce qu'elle apprend. Elle a tendance à prendre des décisions précipitées et à s'attaquer à des faux problèmes.

Afin de mesurer ces styles, Kolb a initialement éléboré puis constamment perfectionné le Learning Style Inventory ${ }^{[11,12]}$, des questionnaires dérivés ayant également été développés.

\section{Objectif de recherche}

Dans le cadre du projet de révision curriculaire précédemment rapporté, la présente étude vise à décrire les styles d'apprentissage des étudiants de la FMS, en se basant sur la typologie inspirée de la théorie d'apprentissage expérientiel.

\section{Sujets et méthodes}

\section{Type d'étude}

Il s'agit d'une étude descriptive, de nature transversale, menée sur une période de trois mois (d'avril à juin 2010), auprès des étudiants de la faculté de médecine de Sousse.

\section{Population étudiée}

Nous avons sollicité la participation des étudiants de cinq promotions successives d'étudiants de la FMS, inscrits respectivement de la première année jusqu'à la cinquième année des études de médecine. La population totale de l'étude se compose de l'ensemble des étudiants de ces différentes promotions, inscrites au cours de l'année universitaire 2009-2010, soit un total de 1043 étudiants. L'identification des personnes devant composer les différents groupes a été effectuée à partir des listes fournies par le service de scolarité de la FMS. Les sollicitations à participer à l'enquête ont été effectuées par contact direct en salles d'enseignements dirigés et lors des stages dans les différents services cliniques. Au cours de ces contacts, l'enquêteur a étayé l'intérêt de l'étude et a pu clarifier le contenu des différents items aux participants en cas de besoin. La collection des questionnaires remplis a eu lieu lors du même contact.

\section{Méthodes}

\section{Choix et description de l'instrument de mesure}

Afin d'identifier les styles d'apprentissage de nos étudiants, nous avons eu recours à un questionnaire développé par l'équipe pluridisciplinaire du laboratoire d'enseignement multimédia (LEM) de l'Université de Liège. Ce questionnaire de 12 situations, appelé "Inventaire des Styles d'Apprentissage du Laboratoire d'Enseignement Multimédia » (ISALEM 97) est basé sur la théorie de l'apprentissage expérientiel de Kolb et le Learning Style Inventory (LSI) du même auteur. Ainsi, le LSI et l'ISALEM 97 sont issus d'un même cadre de référence.

Le LSI a été largement utilisé par de nombreux éducateurs, notamment dans le domaine médical. Bien que ses qualités psychométriques soient considérées comme acceptables, la difficulté exprimée par les répondants à ce questionnaire peut altérer profondément la validité de ses résultats et ainsi la pertinence de son utilisation ${ }^{[13]}$.

Ceci nous a encouragé à exploiter l'ISALEM 97, d'apparition plus récente et en version française, bien que les études rapportant son évaluation soient rares. En effet, la validation psychométrique en a été effectuée par le professeur A. Albert du centre interdisciplinaire de statistique de l'Université de Liège auprès d'un échantillon d'élèves appartenant à l'enseignement 
secondaire général de la communauté francophone de Belgique $^{[14]}$. La validité de construit a été démontrée par le recours à l'analyse factorielle en composantes principales, qui a conclu à la possibilité d'avoir une représentation bidimensionnelle des sujets et des variables. Un peu plus loin dans cet article, nous rapporterons l'analyse factorielle que nous avons obtenue en utilisant ISALEM 97 sur notre propre échantillon.

Concernant la validité convergente, notre revue de littérature ne rapporte pas l'existence d'études démontrant l'existence de corrélation des scores d'ISALEM 97 avec les scores d'autres instruments et évaluant les mêmes concepts. En terme de fidélité (et spécifiquement de consistance interne), l' analyse statistique déjà faite par l'équipe de Liège ne rapporte pas l'étude de l'homogénéité des dimensions calculées. Nous rapporterons plus loin, les coefficients de consistance interne obtenus dans la présente étude.

Par ailleurs, le laboratoire d'enseignement multimédia (LEM) de l'université de Liège publie cet instrument sur son site, ce qui autorise à la communauté scientifique à l'utiliser tout en reconnaissant sa propriété intellectuelle ${ }^{[15]}$.

Ce questionnaire est rédigé en français, ce qui a facilité son administration. Il est formé de 12 situations, pour lesquelles sont proposées chaque fois quatre réactions possibles : « tout à fait moi », « souvent moi », «parfois moi » et « rarement moi ». Chaque étudiant est invité à classer ses réactions dans l'ordre qui correspond le plus à sa préférence.

Une grille de décodage est annexée à ce questionnaire, permettant de calculer les quatre scores suivants, respectivement intitulés : Abstraction -Ab-, Intuition -I -, Action -Ac- et Réflexion -R. Ces scores permettent le calcul de deux facteurs $(\mathrm{X}=\mathrm{I}-\mathrm{Ab}$ et $\mathrm{Y}=$ Ac-R), correspondant respectivement aux deux dimensions étudiées (la saisie et le traitement de l'expérience). Ceci permet de positionner un point dans un référentiel d'axes à deux dimensions, Y en abscisses et $\mathrm{X}$ en ordonnées. Le style d'apprentissage est déterminé en fonction du quadrant dans lequel le point est localisé ${ }^{[15]}$.

Une partie auxiliaire a été rajoutée au questionnaire afin de collecter les caractéristiques sociodémographiques des répondants.

\section{Définition opérationnelle des variables}

Les différents scores calculés correspondent aux différentes étapes du cycle de l'apprentissage expérientiel de Kolb comme suit : l'expérience concrète (intuition), l'observation réfléchie (Réflexion), l'abstraction conceptuelle (Abstraction) et l'expérimentation active (action).

Ainsi, en faisant une analogie avec le LSI, ils peuvent être mis en concordance respectivement avec les quatre styles d'apprentissage de la typologie de Kolb explicitée précédemment ${ }^{[8,13]}$.

Par ailleurs, en se basant sur le fait que l'apprentissage est un processus individuel résultant de l'interaction de l'individu avec l'environnement, outre les style d'apprentissage, certaines caractéristiques socio démographiques peuvent également l'influencer, tels que le genre humain, l'âge ${ }^{[16]}$, d'ou la collecte simultanée de certaines variables socio démographiques.

\section{Analyse des données}

Les données ont été saisies puis exploitées sous SPSS10.0. Les variables quantitatives étaient représentées par la moyenne avec son écart type. Les variables qualitatives ont été décrites avec leurs fréquences absolues et leurs fréquences relatives. Pour les variables suivant une distribution normale, la comparaison de deux moyennes a été effectuée à l'aide du test $t$ de Student. L'analyse de la variance à un critère (ANOVA) a été utilisée pour la comparaison de plusieurs moyennes. Pour les variables ne suivant pas une distribution normale, la comparaison de deux médianes a été effectuée par le test non paramétrique de Mann Whitney et la comparaison de plusieurs médianes a été effectuée par le test de Kruskall Wallis.

La structure factorielle du questionnaire ISALEM 97 a été déterminée sur notre propre population d'étude par une analyse en composantes principales. Les coefficients $\alpha$ de Cronbach correspondant à chaque facteur (ou dimension) ont été calculés. Une matrice de corrélations entre les scores et les facteurs calculés a été également établie.

Tous les résultats étaient considérés comme significatifs au niveau d'incertitude de $5 \%$. 
Tableau I. Profil sociodémographique et éducatif des étudiants de la faculté de médecine de Sousse.

\begin{tabular}{|c|c|c|c|}
\hline \multicolumn{2}{|c|}{ Caractéristiques sociodémographiques et éducatives } & \multirow{2}{*}{\begin{tabular}{|c|} 
Fréquence \\
277 \\
579
\end{tabular}} & \multirow{2}{*}{$\begin{array}{c}\begin{array}{c}\text { Pourcentage } \\
(\%)\end{array} \\
32,4 \\
67,6\end{array}$} \\
\hline Sexe & $\begin{array}{l}\text { - Homme } \\
\text { - Femme }\end{array}$ & & \\
\hline Année d'étude & $\begin{array}{l}\text { - Première année } \\
\text { - Deuxième année } \\
\text { - Troisième année } \\
\text { - Quatrième année } \\
\text { - Cinquième année }\end{array}$ & $\begin{array}{l}225 \\
196 \\
164 \\
117 \\
154\end{array}$ & $\begin{array}{l}26,3 \\
22,9 \\
19,2 \\
13,7 \\
18,0\end{array}$ \\
\hline Niveau de scolarité de la mère & $\begin{array}{l}\text { - N'ayant pas été à l'école } \\
\text { - Primaire } \\
\text { - Secondaire } \\
\text { - Universitaire }\end{array}$ & $\begin{array}{r}35 \\
93 \\
271 \\
457\end{array}$ & $\begin{array}{r}4,1 \\
10,9 \\
31,7 \\
53,4\end{array}$ \\
\hline Niveau de scolarité du père & $\begin{array}{l}\text { - N'ayant pas été à l'école } \\
\text { - Primaire } \\
\text { - Secondaire } \\
\text { - Universitaire }\end{array}$ & $\begin{array}{r}10 \\
45 \\
193 \\
608\end{array}$ & $\begin{array}{r}1,2 \\
5,3 \\
22,5 \\
71,0\end{array}$ \\
\hline Milieu socioéconomique & $\begin{array}{l}\text { - Très en dessous de la moyenne } \\
\text { - Au dessous de la moyenne } \\
\text { - Dans la moyenne } \\
\text { - Au dessus de la moyenne } \\
\text { - Très au dessus de la moyenne }\end{array}$ & $\begin{array}{r}4 \\
20 \\
461 \\
327 \\
44\end{array}$ & $\begin{array}{r}0,5 \\
2,3 \\
53,9 \\
38,2 \\
5,1\end{array}$ \\
\hline Milieu d'origine & $\begin{array}{l}\text { - Rural } \\
\text { - Urbain } \\
\text { - Semi urbain }\end{array}$ & $\begin{array}{r}59 \\
667 \\
130\end{array}$ & $\begin{array}{r}6,9 \\
77,9 \\
15,2\end{array}$ \\
\hline
\end{tabular}

Tableau II. Répartition des étudiants selon l'année d'étude et le sexe.

\begin{tabular}{cccc}
\hline Année de l'étude & Effectif & Age moyen & $\begin{array}{c}\boldsymbol{\%} \text { des filles } \\
\boldsymbol{P}=\mathbf{0 , 4}\end{array}$ \\
\hline $\mathbf{1}^{\text {ère }}$ Année & 225 & $20,0 \pm 0,5$ & $67,6 \%$ \\
\hline $\mathbf{2}^{\text {éme }}$ Année & 196 & $21,1 \pm 0,7$ & $64,3 \%$ \\
\hline $\mathbf{3}^{\text {éme }}$ Année & 164 & $22,2 \pm 0,9$ & $73,8 \%$ \\
\hline $\mathbf{4}^{\text {éme }}$ Année & 117 & $23,5 \pm 1,3$ & $66,7 \%$ \\
\hline $\mathbf{5}^{\text {éme }}$ Année & 154 & $24,5 \pm 1,5$ & $66,2 \%$ \\
\hline
\end{tabular}

\section{Résultats}

Au total 856 étudiants ont répondu au questionnaire, soit $82 \%$ de la population ciblée. La moyenne d'âge des participants était de 21 ans $\pm 0,9$ ans. Le sex-ratio homme/femme était de 0,47 . L'origine urbaine a été notée chez 77,9\% $(n=667)$ des étudiants. Un peu plus de la moitié des étudiants $(53,9 \% ; n=461)$ avait un niveau socio-économique moyen (Tableau I). La prédominance féminine est sensiblement la même dans chaque année d'étude (Tableau II).

Pour chaque étudiant, les quatre scores liés aux différentes étapes du cycle d'apprentissage (Ab, I, Ac et R) ont été étudiés. Les moyennes des scores selon l'année d'étude et selon le sexe sont présentées respectivement dans les tableaux III et IV. L'analyse 
Tableau III. Répartition des moyennes des scores du test ISALEM selon l'année d'étude.

\begin{tabular}{|c|c|c|c|c|c|c|c|}
\hline & $\begin{array}{l}\text { Moyenne } \\
\text { globale }\end{array}$ & 1 ère Année & $\begin{array}{c}2^{\text {éme }} \\
\text { Année }\end{array}$ & $\begin{array}{c}3^{\text {éme }} \\
\text { Année }\end{array}$ & $\begin{array}{c}4^{\text {éme }} \\
\text { Année }\end{array}$ & $\begin{array}{c}5^{\text {éme }} \\
\text { Année }\end{array}$ & $p$ \\
\hline $\begin{array}{l}\text { Abstraction } \\
(\mathrm{M} \pm \mathrm{ET})\end{array}$ & $27,4 \pm 5,1$ & $27,8 \pm 5,0$ & $27,2 \pm 4,9$ & $27,9 \pm 5,1$ & $26,6 \pm 4,6$ & $27,2 \pm 5,5$ & $p=0,1$ \\
\hline $\begin{array}{l}\text { Intuition } \\
(\mathrm{M} \pm \mathrm{ET})\end{array}$ & $32,3 \pm 4,6$ & $32,3 \pm 4,7$ & $32,3 \pm 4,5$ & $31,7 \pm 4,6$ & $32,7 \pm 4,0$ & $32,5 \pm 5,2$ & $p=0,5$ \\
\hline $\begin{array}{l}\text { Action } \\
(\mathrm{M} \pm \mathrm{ET})\end{array}$ & $32,7 \pm 4,4$ & $32,2 \pm 4,5$ & $32,6 \pm 4,4$ & $32,7 \pm 4,1$ & $33,2 \pm 4,3$ & $32,9 \pm 4,6$ & $p=0,3$ \\
\hline $\begin{array}{l}\text { Réflexion } \\
(\mathrm{M} \pm \mathrm{ET})\end{array}$ & $27,5 \pm 4,4$ & $27,5 \pm 4,4$ & $27,7 \pm 4,4$ & $27,5 \pm 4,5$ & $27,3 \pm 4,1$ & $27,2 \pm 4,3$ & $p=0,9$ \\
\hline
\end{tabular}

M = moyenne

ET = écart type

ISALEM = Inventaire des Styles d'Apprentissage du Laboratoire d'Enseignement Multimédia.

Tableau IV. Répartition des moyennes des scores du test ISALEM selon le genre.

\begin{tabular}{lccc}
\hline & Homme & Femme & $p$ \\
\hline Abstraction (M \pm ET) & $27,53 \pm 5,23$ & $27,04 \pm 5,06$ & 0,72 \\
\hline Intuition (M \pm ET) & $31,9 \pm 4,95$ & $32,5 \pm 4,54$ & 0,11 \\
\hline Action $(\mathbf{M} \pm \mathbf{E T})$ & $32,04 \pm 4,74$ & $33,03 \pm 4,29$ & 0,002 \\
\hline Réflexion $(\mathbf{M} \pm \mathbf{E T})$ & $28,47 \pm 4,41$ & $27,04 \pm 4,33$ & $<10^{-3}$ \\
\hline
\end{tabular}

M : Moyenne de la variable étudiée

ET : Écart type

$p$ : degré de significativité

ISALEM = Inventaire des Styles d'Apprentissage du Laboratoire d'Enseignement Multimédia.

statistique ne montre pas de différence significative selon l'année d'étude. Par contre, on note une différence selon le sexe au niveau des scores de réflexion et d'action. En effet, comparativement aux garçons, les filles avaient un score plus élevé pour l'action (33 contre $32 ; p=0,002)$ et un score moins important pour la réflexion (27 contre 28,$4 ; p \leq 10^{-3}$ ).

Les quatre scores correspondant aux différentes étapes du cycle sont composés de deux dimensions dialectiquement indépendantes et correspondent aux deux facteurs $\mathrm{X}$ et $\mathrm{Y}$. Le facteur $\mathrm{X}$, constitué à partir des scores opposés d'abstraction et d'intuition, reflète la dimension de saisie de l'expérience. De même, le facteur Y, constitué à partir des scores d'action et de réflexion, représente la dimension de traitement de l'expérience. Les médianes de ces facteurs sont représentées dans les tableaux V et VI, respectivement en fonction de l'année d'étude et du sexe. Nous n'avons pas noté de différence significative pour le facteur $\mathrm{X}$ ni en fonction du genre ni pour l'année de l'étude. Par contre, il a été observé une différence en fonction du sexe pour la dimension de traitement de l'information (Y).

La description du style d'apprentissage des étudiants a montré que la majorité des étudiants (40\%, $n=314$ ) avaient une préférence pour le style intuitif pragmatique (accommodateur) (Figure 2). Vingt huit pour cent des sujets $(n=220)$ avaient plutôt un style méthodique pragmatique (convergent) et 24,2\% ( $n=$ 190) un style méthodique réflexif (assimilateur). Par contre seulement 7,5\% $(n=59)$ avaient un style de type intuitif réflexif (divergent).

Les analyses en composantes principales confirment la structure bidimensionnelle du questionnaire 
Tableau V. Répartition des médianes des facteurs du test ISALEM selon l'année d'étude.

\begin{tabular}{lcccccc}
\hline & $\mathbf{1}^{\text {ère }}$ Année & $\mathbf{2}^{\text {éme }}$ Année & $\mathbf{3}^{\text {éme }}$ Année & 4 $^{\text {éme }}$ Année & 5 éme $^{\text {Année }}$ & $\boldsymbol{p}$ \\
\hline $\mathbf{X}=\mathbf{I}-\mathbf{A b}$ & $-4[-33 ; 17]$ & $-4[-34 ; 19]$ & $-5[-26 ; 14]$ & $-2[-30 ; 13]$ & $-2[-35 ; 18]$ & 0,19 \\
$(\mathrm{Me} ;[\mathbf{m i n} ; \mathbf{m a x}])$ & & & & & & \\
\hline $\mathbf{Y}=\mathbf{A c}-\mathbf{R}$ & $0[-22 ; 20]$ & $0[-24 ; 19]$ & $0[-21 ; 16]$ & $2[-18 ; 20]$ & $2[-23 ; 19]$ & 0,52 \\
$(\mathrm{Me} ;[\mathbf{m i n} ; \max ])$ & & & & & \\
\hline
\end{tabular}

Me : Médiane de la variable étudiée

[Min ; max] : Minimum ; Maximum

$p$ : degré de significativité

ISALEM = Inventaire des Styles d'Apprentissage du Laboratoire d'Enseignement Multimédia.

Tableau VI. Répartition des médianes des facteurs $X$ (saisie de l'expérience) et $Y$ (traitement de l'expérience) en fonction du sexe.

\begin{tabular}{lccc}
\hline & Homme & Femme & $p$ \\
\hline $\mathbf{X}=\mathbf{I}-\mathbf{A b}$ & $-4[-35 ; 18]$ & $-4[-34 ; 19]$ & 0,4 \\
$(\mathbf{M e} ;[\mathbf{m i n} ; \mathbf{m a x}])$ & & & $<10^{-3}$ \\
\hline $\mathbf{Y}=\mathbf{A c}-\mathbf{R}$ & $-1[-23 ; 19]$ & $2[-24 ; 20]$ & \\
$(\mathbf{M e} ;[\mathbf{m i n} ; \mathbf{m a x}])$ & & & \\
\hline
\end{tabular}

Me : Médiane de la variable étudiée

[Min ; max ] : Minimum ; Maximum

$p:$ degré de significativité.

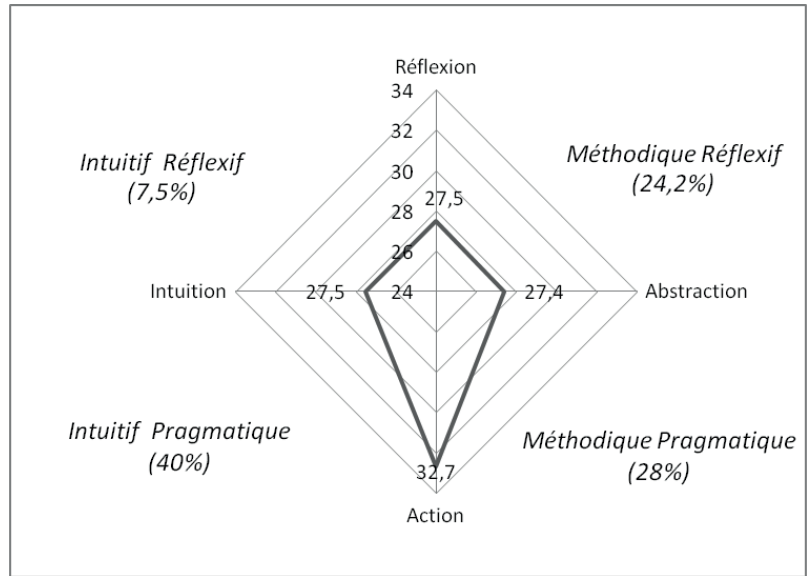

Fig. 2. Répartition des étudiants de la faculté de médecine de Sousse selon les styles d'apprentissage.

ISALEM 97. En effet, le premier facteur explique à lui seul $58,6 \%$ de la variance totale des scores et les deux facteurs cumulés en expliquent $81,2 \%$.

$\mathrm{Au}$ regard de la consistance interne du questionnaire ISALEM 97, dans notre population, nous avons obtenu un coefficient de Cronbach de 0,78 pour la dimension « Saisie de l'information » $(\mathrm{X})$ et de 0,72 pour la dimension « Traitement de l'information » (Y) (voir Tableau VII).

Le tableau VIII rapporte les résultats des corrélations inter scores et inter facteurs. Ces corrélations sont dans la majorité inférieures à 0,6 ce qui signifie l'absence de redondance entre les scores et les facteurs, sauf pour la corrélation entre le score d'abstraction et le score d'intuition $(r=-0.65)$. Par ailleurs, des corrélations négatives ont été identifiées entre les scores de mesure des constructions bipolaires : abstraction versus Intuition $(r=-0.65)$ et action versus réflexion $(r=-0,57)$. Une corrélation positive entre les deux facteurs étudiés X et Y $(r=$ 0,45) a été observée.

\section{Discussion}

Ce travail a été motivé par le processus général de réforme pédagogique institutionnelle initié à la Faculté de médecine de Sousse, avec l'intention d'améliorer la qualité des apprentissages des étudiants et, ce 
Tableau VII. Descriptif et cohérence interne des facteurs validés de l'ISALEM 97.

\begin{tabular}{lcccc}
\hline Facteurs & $\begin{array}{c}\text { Nombre } \\
\text { d'observations }\end{array}$ & M & ET & $\alpha$ \\
\hline $\mathrm{X}=\mathrm{I}-\mathrm{Ab}$ & 856 & 4,8 & 8,9 & 0,78 \\
\hline $\mathrm{Y}=\mathrm{Ac}-\mathrm{R}$ & 856 & 5,2 & 7,8 & 0,72 \\
\hline $\mathrm{M}:$ Moyenne de la variable étudiée & & & \\
$\mathrm{ET}:$ Écart type & & & \\
$\alpha:$ Coefficient Cronbach & & &
\end{tabular}

ISALEM = Inventaire des Styles d'Apprentissage du Laboratoire d'Enseignement Multimédia.

Tableau VIII. Validité du contenu et redondance : matrice de corrélation entre les scores et les facteurs étudiés.

\begin{tabular}{|c|c|c|c|c|c|c|}
\hline & $\mathbf{A b}$ & I & Ac & $\mathbf{R}$ & $\mathbf{I}-\mathbf{A b}$ & $\mathbf{A c}-\mathrm{R}$ \\
\hline $\mathbf{A b}$ & - & & & & & \\
\hline \multirow[t]{2}{*}{$\mathbf{I}$} & $r=-0,65$ & - & & & & \\
\hline & $p<10^{-4}$ & & & & & \\
\hline \multirow[t]{2}{*}{ Ac } & $r=-0,58$ & $r=0,22$ & - & & & \\
\hline & $p<10^{-4}$ & $p<10^{-4}$ & & & & \\
\hline \multirow[t]{2}{*}{$\mathbf{R}$} & $r=0,12$ & $r=-0,52$ & $r=-0,57$ & - & & \\
\hline & $p<10^{-4}$ & $p<10^{-4}$ & $p<10^{-4}$ & & & \\
\hline \multirow[t]{2}{*}{$\mathbf{I}-\mathbf{A b}$} & $r=-0,91$ & $r=0,9$ & $r=0,45$ & $r=-0,34$ & - & \\
\hline & $p<10^{-4}$ & $p<10^{-4}$ & $p<10^{-4}$ & $p<10^{-4}$ & & \\
\hline \multirow[t]{2}{*}{$A c-R$} & $r=-0,39$ & $r=0,42$ & $r=0,88$ & $r=-0,88$ & $r=0,45$ & - \\
\hline & $p<10^{-4}$ & $p<10^{-4}$ & $p<10^{-4}$ & $p<10^{-4}$ & $p<10^{-4}$ & \\
\hline
\end{tabular}

$r:$ coefficient de corrélation de Pearson

$p:$ degrés de significativité.

faisant, d'apporter une contribution à l'amélioration des compétences des futurs diplômés, grâce à une identification et une meilleure prise en compte de leurs styles d'apprentissage.

Pour répondre à cet objectif, nous nous sommes appuyés sur le cadre conceptuel de l'apprentissage expérientiel de Kolb. Cette théorie postule que l'apprentissage est le processus par lequel le savoir est créé par la transformation de l'expérience ${ }^{[8,9]}$. Elle se prête en effet à notre domaine d'étude (les études médicales), dont le contexte est celui d'un processus continu d'apprentissage fortement ancré sur l'exposition des apprenants à des expériences professionnelles.

En assumant une analogie des styles d'apprentissage mesurés par le questionnaire ISALEM 97 avec ceux de la typologie de Kolb, mesurés par le test LSI $^{[12]}$, notre étude montre que les étudiants de notre institution ont dans $40 \%$ des cas un style intuitifpragmatique (accommodateur), dans $28 \%$ un style méthodique-pragmatique (convergent), dans $24,2 \%$ un style méthodique-réflexif (assimilateur) et dans $7,5 \%$ un style intuitif-réflexif (divergent). Ils sont ainsi assez comparables aux étudiants pakistanais étudiés par Danich et Awan, chez qui prédominait également le style accommodateur ${ }^{[17]}(54,6 \%)$, mais ils se distinguent de ceux de l'étude d'Engels et Gara ${ }^{[18]}$, qui concluait à une prépondérance du style assimilateur $(40 \%$ des étudiants en médecine de l'Université d'Alberta), de ceux de l'étude de Gurpinar et al. ${ }^{[19]}$ où le style divergent était prédominant $(47,7 \%$ des étudiants de première année d'une université turque) et de ceux de l'étude nord-américaine de Chapman et Calhoun $^{[2]}$, qui étaient plutôt de style abstrait et actif. Nos étudiants privilégiaient ainsi l'action guidée par l'intuition plutôt que l'analyse logique. Ce profil 
prédominant de nos étudiants semble différent de ce que pouvait laisser prévoir la littérature au regard des caractéristiques du contexte médical. En effet, parmi les cinq facteurs pouvant influencer le style d'apprentissage, Kolb ${ }^{[12]}$ cite la spécialisation éducative de l'apprenant : les personnes spécialisées dans les arts, les sciences politiques, les langues étrangères et la psychologie ont tendance à adopter un style divergent, tandis que ceux spécialisés dans des domaines plus abstraits tels que la médecine et l'ingénierie sont plutôt convergents. Les personnes ayant un style accommodateur préfèrent plutôt les sciences d'éducation et de la communication et ceux du style assimilateur plutôt les mathématiques et les sciences physiques.

L'étude des dimensions d'apprentissage ne varie pas selon l'année d'étude. Cependant, on a noté une différence selon le sexe au niveau de la transformation de l'expérience. Ainsi, les filles ne différent pas des garçons en ce qui concerne la saisie de l'expérience à partir de l'intuition ou de l'abstraction. Par contre, elles ont davantage de capacité à tester l'application des concepts à des nouvelles situations, alors que les garçons donnent plus de l'importance à l'observation et à la réflexion. Dans la littérature, certaines études montrent que le style d'apprentissage diffère selon le sexe $;$ Keri $^{[20]}$ rapporte ainsi que les filles ont un style plutôt abstrait et conceptuel alors que les garçons sont plutôt concrets et pratiques.

Certains aspects psychométriques de notre travail méritent d'être discutés. Nous avons précédemment argumenté le choix du questionnaire ISALEM 97, en raison de son caractère plus récent, de sa rédaction en langue française et de sa structure bidimensionnelle. Notre étude contribue à documenter la validité et la fidélité de cet instrument. Concernant sa validité de construit, les analyses factorielles en composantes principales accréditent la bi-dimensionnalité du questionnaire. Concernant la validité du contenu, nos résultats sont cohérents avec les prévisions des corrélations inter scores et inter facteurs établies par $\mathrm{Kolb}^{[12]}$. En effet, les scores opposés d'une même dimension se trouvent négativement corrélés, avec des coefficients $r$ de Pearson proches de 0,6. Ceci peut être expliqué par le fait que la relation dialectique prévoit la possibilité d'intégration des pôles opposés ${ }^{[12]}$. Concernant la fidélité et notamment la dimension de consistance interne, les coefficients $\alpha$ de Cronbach calculés à partir de nos résultats sont acceptables, bien qu'on ignore l'évaluation de la consistance interne faite par l'équipe de Liège.

Certaines limites de notre étude doivent enfin être mentionnées. En effet, l'objectif de l'enquête n'a pas été bien saisi par certains étudiants et le test préalable du questionnaire n'a pas empêché de constater que certains termes n'ont pas été bien compris, malgré une administration du questionnaire assistée par un enquêteur afin de clarifier l'objectif ainsi que certains items. Par ailleurs, si l'exhaustivité de la population de l'étude avec un taux de participation assez important est à mettre au crédit de sa représentativité de la population pour la faculté de médecine de Sousse, l'extrapolation des résultats aux étudiants des autres facultés de médecine en Tunisie pourraitêtre discutée, de sorte que la validité externe de notre étude ne peut être tenue pour acquise, alors même que l'univocité des recommandations concernant les programmes d'enseignement et les orientations pédagogiques, telles qu'elles sont formulées par les facultés tunisiennes, plaiderait pour que ces résultats soient généralisables.

Cependant, la discussion la plus importante relative à notre travail est d'une autre nature. Elle concerne finalement l'importance de la nécessité qu'il y aurait de se préoccuper explicitement des styles d'apprentissage des étudiants et d'y adapter les dispositifs pédagogiques. Lorsque ce travail a été entrepris, au décours de la démarche d'évaluation institutionnelle effectuée de 2003 à 2005, cette préoccupation paraissait raisonnablement légitime, sur la foi de l'étude exploratoire de la littérature disponible à l'époque. Cependant, une analyse plus attentive des écrits révèle que seules quelques études accréditent l'hypothèse qu'un enseignement plus personnalisé et centré sur les styles d'apprentissage favorise effectivement de meilleurs apprentissages. À partir de 2009, des réflexions critiques à l'égard de l'importance de la notion de style d'apprentissage ont commencé à apparaître dans la littérature en éduction médicale. Ainsi, commentant dans un éditorial les résultats d'une étude qui montrait l'absence d'effet d'un dispositif pédagogique visant à prendre en compte les styles d'apprentissage ${ }^{[21]}$, Norman ${ }^{[22]}$ 
conclut radicalement que la notion de style d'apprentissage serait obsolète. Dans un autre commentaire éditorial plus récent, Rohrer et Pashler ${ }^{[23]}$ précisent que seules vingt études ont évalué l'impact d'une approche d'enseignement adaptée au style d'apprentissage des étudiants et que la majorité d'entre elles, incluant celles conduites dans le domaine médical et scientifique, ont donné des résultats négatifs. L'une des explications pourrait être que ce type d'enseignement comporte certaines exigences logistiques, liées par exemple à la nécessité d'élaborer le cours sous différentes versions afin de satisfaire tous les styles d'apprentissages ou encore à celle de développer des dispositifs d'évaluation personnalisés des apprentissages des étudiants par des tests parfois coûteux. Les auteurs considèrent qu'au regard de l'état de la recherche, il est aujourd'hui plus judicieux de porter ses efforts sur le caractère varié et éclectique des ressources didactiques fournies aux étudiants, plutôt que de chercher à tout prix à développer des interventions pédagogiques qui seraient ajustées «sur mesure » aux styles d'apprentissage des étudiants.

\section{Conclusion}

Au total, les résultats de notre étude montrent que les étudiants de la faculté de médecine de Sousse recourent majoritairement à un style d'apprentissage de type intuitif-pragmatique (accommodateur) ; ils recourent à l'intuition pour la saisie des concepts et ont tendance à les appliquer à de nouvelles expériences pour résoudre les problèmes. Ce profil ne change pas tout au long de leurs années d'études médicales mais diffère légèrement en fonction du sexe. La nécessité de prendre systématiquement en compte les styles d'apprentissage des étudiants dans le cadre de dispositifs pédagogiques spécifiques fait cependant actuellement l'objet de discussions critiques.

\section{Contributions}

Meriam El Ghardallou et Ali Mtiraoui ont contribué à la conception du protocole de recherche de l'étude. Meriam El Ghardallou, Thouraya Ajmi et
Iheb Bougmiza ont rédigé l'article. Meriam El Ghardallou et Iheb Bougmiza ont analysé et interprété les résultats. Chekib Zedini et Manel Mellouli ont participé au recueil et à la saisie des données. Ali Mtiraoui était le chef des travaux de l'unité de recherche au Département de médecine familiale et communautaire à la Faculté de médecine de Sousse. Meriam El Ghardallou, Thouraya Ajmi, Iheb Bougmiza et Ali Mtiraoui ont révisé le manuscrit suite aux suggestions des lecteurs experts et du comité de rédaction de la revue.

\section{Approbation éthique}

Le projet de recherche n'a pas été examiné par un comité d'éthique, cette exigence n'étant pas institutionnellement requise au moment de l'étude. Le consentement informé des étudiants a été systématiquement recherché et obtenu oralement dans le cadre d'un entretien.

\section{Déclaration d'intérêt}

Les auteurs ne déclarent aucun conflit d'intérêt en lien avec la problématique de l'étude.

\section{Références}

1. Projet d'établissement: Plan stratégique 2009-2013: Ministère de l'Enseignement Supérieur, de la Recherche Scientifique et de la Technologie,. Sousse : Université de Sousse, Faculté de Médecine de Sousse, 2005.

2. Chapman DM, Calhoun JG. Validation of learning style measures: implications for medical education practice. Med Educ 2006;40:576-83.

3. Samarakoon L, Fernando Th, Rodrigo Ch, Rajapakse S. Learning styles and approaches to learning among medical undergraduates and postgraduates. BMC Medical Education 2013;13(42).

4. Côté D, Graillon A, Waddell G, Lison Ch, Noel MF. L'approche d'apprentissage dans un curriculum 
médical préclinique basé sur l'apprentissage par problèmes. Pédagogie Médicale 2006;7:201-12.

5. Biggs J. Student approaches to learning and studying. Hawthorn, Vic.: Australian Council for Educational Research, 1987.

6. Kirby JR, Woodhouse RA. Measuring and predicting depth of processing in learning. Alberta J Educ Res 1994;40:147-61.

7. Allport G. Pattern and Growth in Personality. New York : Holt, Rinehart and Winston, 1961.

8. Chevrier J, Fortin G, Théberge M, Leblanc R. Le style d'apprentissage: une perspective historique. Revue scientifique virtuelle: Education et Francophonie 2000;18:20-46.

9. Kolb DA. Experiential Learning : Experience as the Source of Learning and Development. Englewood Cliffs, New Jersey : Prentice-Hall, 1984.

10. Bernard JL, Reyes P. Apprendre, en médecine ( $1^{\text {ère }}$ partie). Pédagogie Médicale 2001;2:163-9.

11. Kolb DA. LSI - Learning Style Inventory: Technical Manual. Boston (MA) : McBer, 1976

12. Kolb AY, Kolb D. The Kolb Learning Style InventoryVersion 3.1. Technical Specifications. London: Hay Resources Direct, 2005.

13. Cook DA, Smith AJ. Validity of index of learning styles scores: multitrait- multimethod comparison with three cognitive / learning style instruments. Med Educ 2006;40:900-7.

14. Albert A. Le questionnaire ISALEM : étude Statistique. Centre interdisciplinaire de Statistique de l'Université de Liège. [On-line] Disponible sur : http://www2.ulg.ac.be/lem/StyleApprent/ StyleApprent_CG/media/isalem_stat_compact.pdf

15. Cahay R, Honorez M, Monfort B, Remy F, Therer J. Comment identifier les styles d'apprentissage : ISALEM-97. Laboratoire d'Enseignement Multimédia
(LEM) de l'Université de Liège. 1997 [On-line] Disponible sur : http://www2.ulg.ac.be/lem/StyleApprent/StyleApprent_CG/page_05.htm

16. Bayrak BK, Altun S. Is there any differnce between learning styles of student science teachers in relation to both their grade and gender ? Procedia - Social and Behavioral Sciences 2009:765-70.

17. Danich K, Awan A. A study of students' learning style in a medical institution in Pakistan. Rawal Med Journal 2008;33:239-41.

18. Engels P, De Gara Ch. learning styles of medical students, genral syrgry residents, and general surgerons: implications for surgical education. BMC Medical Education 2010;10(51).

19. Gurpinar E, Alimoglu M, Mamakli S, Aktekin M. Can learning style predict student satisfaction with different instruction methods and academic achievement in medical education? Adv Physiol Educ 2010; 34:192-6.

20. Keri G. Male and female college students' learning styles differ: An opportunity for instructional diversification. College Student Journal 2002;36:433-42.

21. Cook DA, Thompson WG, Thomas KG, Thomas MR. Lack of interaction between sensing-intuitive learning styles and problem-first versus information-first instruction: a randomized crossover trial. $\mathrm{Adv}$ in Health Sci Educ Theory Pract 2009;14:79-90.

22. Norman G. When will learning style go out of style? Adv in Health Sci Educ Theory Pract 2009;14:1-4.

23. Rohrer D, Pashler H. Learning styles: Where's the evidence? Med Educ 2012;46:630-5.

Correspondance et offprints : Meriam El Ghardallou, Département de médecine familiale et communautaire, Faculté de médecine Ibn El Jazzar, Avenue Mohamed Karoui, 4000 Sousse, Tunisie.

Mailto : meriamelghardallou@yahoo.fr 